\title{
A Subpixel Edge Detector Applied to Aortic Dissection Detection
}

\author{
A. Trujillo-Pino ${ }^{1}$, K. Krissian ${ }^{1}$, D. Santana-Cedrés ${ }^{1}$, J. Esclarín-Monreal ${ }^{1}$, \\ and J.M. Carreira-Villamor ${ }^{2}$ \\ 1 Centro de Tecnologías de la Imagen (CTIM) \\ Universidad de Las Palmas de Gran Canaria (ULPGC)* \\ 2 Universidad de Santiago de Compostela (USC) \\ \{agustin,krissian, jesclarin\}@dis.ulpgc.es, dsantana@ctim.es, \\ josemartin.carreira@usc.es
}

\begin{abstract}
The aortic dissection is a disease that can cause a deadly situation, even with a correct treatment. It consists in a rupture of a layer of the aortic artery wall, causing a blood flow inside this rupture, called dissection. The aim of this paper is to contribute to its diagnosis, detecting the dissection edges inside the aorta. A subpixel accuracy edge detector based on the hypothesis of partial volume effect is used, where the intensity of an edge pixel is the sum of the contribution of each color weighted by its relative area inside the pixel. The method uses a floating window centred on the edge pixel and computes the edge features. The accuracy of our method is evaluated on synthetic images of different thickness and noise levels, obtaining an edge detection with a maximal mean error lower than 16 percent of a pixel.
\end{abstract}

Keywords: Aortic Dissection, Subpixel Edge Detection, Anisotropic Diffusion.

\section{Introduction}

\subsection{The Aortic Dissection}

To understand the aortic dissection, we need to know the artery structure. The wall of an artery is formed by three layers, from inside to outside: the tunica intima, the tunica media and the tunica adventitia (fig. 1, left).

Under normal conditions, the blood flows inside the vessel lumen (fig. 1, center). But, in the case of an aortic dissection, the normal blood flow changes. The tunica intima breaks and the blood gets into the tunica media (fig. 1, right). Therefore, we have two flows: the normal flow inside the vessel lumen, and the flow inside the tunica media, called false vessel lumen.

At the beginning of the generation process of an aortic dissection, we have a normal flow. When the dissection occurs, in most cases, there are one or two

\footnotetext{
* This work has been supported by the project SIMVA, TIN2009-10770 from the Spanish Ministry of Science and Innovation.
} 

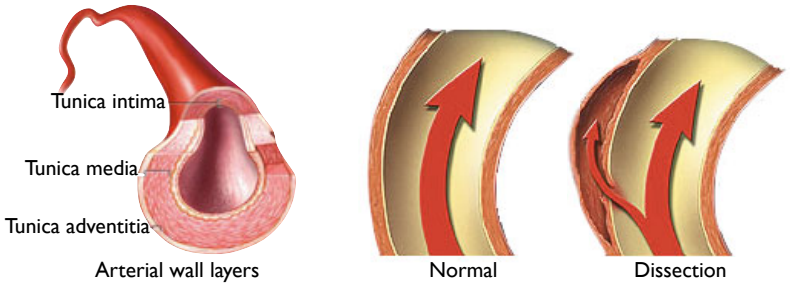

Fig. 1. Artery structure (Courtesy of A.D.A.M. Inc.) and Normal vs Dissection blood flow (Courtesy of Dr. Grasshopper)

tears (also called entry points) that connect with the vessel lumen. At this moment the false vessel lumen is created (extraluminal wall channel). A dissection may have a variable separation, length and thickness (from few millimeters to several centimeters). When there are no more entry points, we find an intramural hematoma, that can progress to a complete dissection, through a secondary rupture of the tunica media.

\subsection{Aortic Dissection Classification}

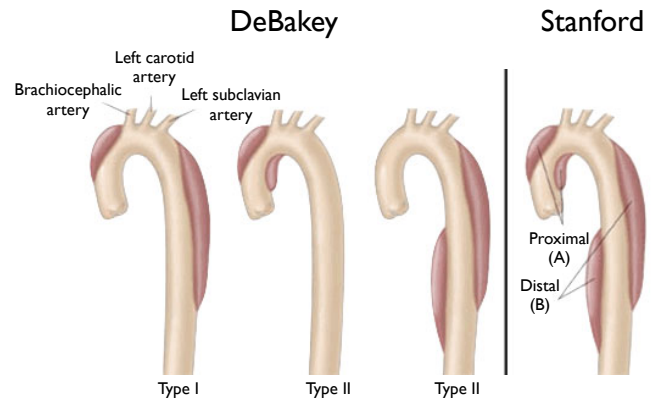

Fig. 2. DeBakey and Stanford aortic dissection classification (Courtesy of Richard J. Shemin, MD; Praveen Menon,MD; Ara Ketchedjian, MD Medscape Education (Medscape Cardiology))

There are several types of classification, based on the starting point, the type and length of the injury and the symptoms duration. Initially, the DeBakey classification was used. It was defined by Dr. Michael Ellis DeBakey, a worldrenowned cardiovascular surgeon and scientist 2. It divides the dissection in three types (fig. 2, left): Type I, type II and type III (IIIA or IIIB depending on the dissection end location).

But now, the Stanford classification, based on the prognosis differences and the therapeutic treatment, is the standard one. It divides the dissection in two 
types (fig. 2, right): Type A (starts at ascending aorta and can be extended to aortic arch and descending thoracic aorta) and type B (everyone else).

Based on the Stanford classification, we have the following statistics: from 58 to 72 percent of the dissections are of type A, and from 28 to 42 percent are of type B. Moreover, 70 percent of the dissections end with a rupture of the artery wall, with a bled on adjacent structures, such as pericardial bled (more frequent on type A) [1.

\section{$1.3 \quad$ Diagnostic Images}

Angiography has been replaced now by other imaging techniques, such as computed tomography (CT) or magnetic resonance (MRI). In the fig. 3 we can see the axial, coronal and sagittal plane of a $\mathrm{CT}$; with a zoom on the dissection region. The arrow indicates the location of the aortic dissection.
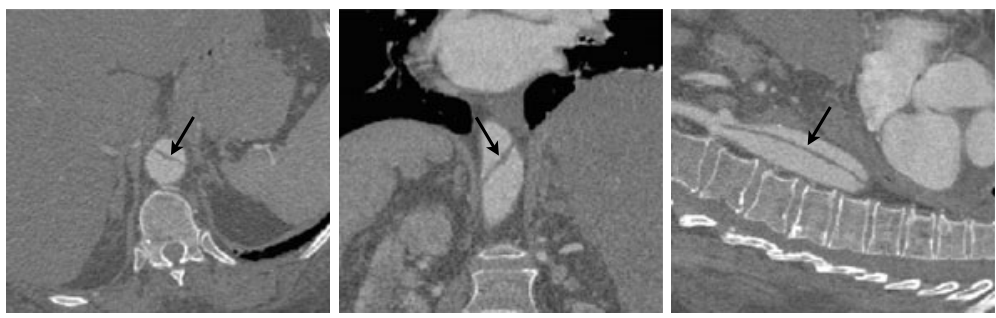

Fig. 3. Axial, coronal and sagittal plane of an aortic dissection. The black arrow indicates the location of the dissection inside the aorta.

To contribute to the diagnosis of the medical problem, the dissection must be located. The correct estimation of edge features is an important step to extract higher-level information. The subpixel edge detector proposed by Agustín Trujillo Pino[7, uses a floating window to calculate the edge features with high accuracy.

We propose to use this subpixel edge detector for locating the aortic dissection edges, because it has a thin planar structure, that divides the interior of the aorta in two or more parts. For the experiments, we use a synthetic model of the structure, and apply the method to different configurations of thickness and noise.

\section{Method}

\subsection{The Subpixel Method}

An edge is a virtual line between two regions with different intensity levels. Normally, the standard methods only indicate the edge pixels. They usually use numerical information of the pixels of a particular neighborhood to decide if it 
is an edge pixel or not. However, when accuracy is important, for example when measuring the dissection thickness; a pixel level detection isn't appropriate. A subpixel edge detection must be used.

The subpixel edge detection method proposed by Trujillo [7, is based on the partial volume effect inside the edge pixels. Most methods assume that an image is a continuous and differentiable function. The subpixel edge detection method assumes that an edge is a discontinuity in the intensity values of the function, delimiting the border between two objects. The intensity of an edge pixel is the sum of the contribution of each value, weighted by its relative area inside the pixel. Other methods apply any of the following techniques: moment [3], least squared [4] or interpolation [6]. The main advantage of this subpixel method is that all the edge features (sub-pixel position, orientation, curvature and change in intensity at both sides) are computed with total accuracy in ideal images.

At the beginning, the subpixel algorithm smooths the input image using a $3 \times 3$ mask obtaining the image $G$. Afterwards x and y derivatives are calculated at the $(i, j)$ pixel, and according to the maximum absolute value, the orientation (vertical or horizontal) of the window centered in this pixel is decided. Once known that the partial value is greater than the threshold, the method computes the limits of the floating window (fig. 4). In the vertical case, the window width is constant, three pixels, and the maximum height is nine pixels. The height limits of the window are set to be the pixels of minimal derivative values on each side of the center pixel within each column or row of nine pixels. The use of the floating limits allows to detect close-by edges (separated by four pixels or more).

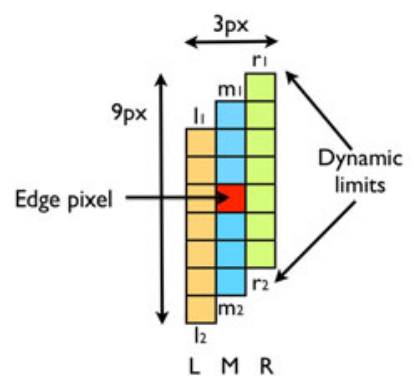

Fig. 4. Floating window of the subpixel edge detector

In the next step, the method computes the intensity values at both sides of the edge (eq. 1 and 2). This calculation is done considering the sign of the product between $\mathrm{x}$ and $\mathrm{y}$ derivatives. In all cases, the pixels used to compute the intensity are indicated by the floating limits computed before $\left(l_{1}, l_{2}, m_{1}, m_{2}, r_{1}, r_{2}\right)$.

$$
B=\left\{\begin{array}{l}
\frac{G_{i-1, j+l_{1}}+G_{i, j+m_{1}}}{2} \text { if } G_{x}(i, j) G_{y}(i, j)>0 \\
\frac{G_{i, j+m_{1}}+G_{i+1, j+r_{1}}}{2} \text { if } G_{x}(i, j) G_{y}(i, j)<0
\end{array}\right.
$$




$$
A=\left\{\begin{array}{l}
\frac{G_{i, j+m_{2}}+G_{i+1, j+r_{2}}}{2} \text { if } G_{x}(i, j) G_{y}(i, j)>0 \\
\frac{G_{i-1, j+l_{2}+G_{i, j+m_{2}}}}{2} \text { if } G_{x}(i, j) G_{y}(i, j)<0
\end{array}\right.
$$

There are cases where the edges are very close. For example, the dissection region could have a thickness of two or three pixels. The subpixel method can detect this type of edges from the derivative values after the limits. If these values are very high, we will be in this case, and the value of the intensity will be computed using the original image.

The last step is computing the sum of every column. This is possible using the floating limits computed in previous steps (eq. 3). With the values of the intensity at both sides of the edge pixel and the sums of the columns, we can compute the parameters of the edge. The method finds the coefficients of the parabola $\left(y=a+b x+c x^{2}\right)$ which best fits the edge (eq. 4). Applying these steps to every edge pixel, the edges of the image are obtained with subpixel accuracy. The values of $a, b$ and $c$ are obtained from as follows:

$$
\begin{aligned}
S_{L} & =\sum_{k=l_{1}}^{l_{2}} G_{i-1, j+k} \quad S_{M}=\sum_{k=m_{1}}^{m_{2}} G_{i, j+k} \quad S_{R}=\sum_{k=r_{1}}^{r_{2}} G_{i+1, j+k} \\
c & =\frac{S_{L}+S_{R}-2 S_{M}}{2(A-B)}+\frac{A\left(2 m_{2}-l_{2}-r_{2}\right)-B\left(2 m_{1}-l_{1}-r_{1}\right)}{2(A-B)} \\
b & =\frac{S_{R}-S_{L}}{2(A-B)}+\frac{A\left(l_{2}-r_{2}\right)-B\left(l_{1}-r_{1}\right)}{2(A-B)} \\
a & =\frac{2 S_{M}-\left(1+2 m_{2}\right) A-\left(1-2 m_{1}\right) B}{2(A-B)}-\frac{1+24 a_{01}+48 a_{11}}{12} c
\end{aligned}
$$

\subsection{Noise Reduction}

When the input image is noisy, it must be filtered previously to better detect the edges. For this purpose, the Noise Reducing Anisotropic Diffusion filter (NRAD) [5] is used. The NRAD method filters the noise preserving the edges, like Perona and Malik method:

$$
\left\{\begin{array}{l}
u(0)=u_{0} \\
\frac{\partial u}{\partial t}=\operatorname{div}(c \nabla u)
\end{array}\right.
$$

This equation (5) uses a diffusion coefficient, that is a decreasing function of the gradient norm. This diffusion function depends on a parameter related to the image gradient that must be set by the user. However, the NRAD filter (eq. 66), uses a diffusion function $1-k$, that depends on the local statistics of the image and on the noise model.

$$
\left\{\begin{array}{l}
u(0)=u_{0} \\
\frac{\partial u}{\partial t}=\operatorname{div}((1-k) \nabla u)=\operatorname{div}\left(\frac{\sigma_{n}^{2}}{v_{g}} \nabla u\right)
\end{array}\right.
$$

An additive noise model is used in the experiments, $g=f+n$, where the observed image, $g$, is equal to the sum of the ideal image, $f$, plus a Gaussian additive noise, $n$. 
Equation 7 shows that the corrected image $\widehat{f}$ is calculated using an estimator, and it is the sum of the local mean $\bar{g}$ and the product between the coefficient $k$ and the difference between the image $g$ and its local mean $\bar{g}$.

The coefficient $k$ (eq. 8 ) is the result of the division between the local variance of $\mathrm{f}, v_{f}$, and the local variance of $\mathrm{g}, v_{g}$. The parameter $\sigma_{n}^{2}$ is the global noise variance, computed at every iteration using a region of interest. The partial differential equation (6) is based on this estimator.

$$
\begin{aligned}
& \widehat{f}=\bar{g}+k(g-\bar{g}) \\
& k=\frac{v_{f}}{v_{g}}=\frac{v_{g}-\sigma_{n}^{2}}{v_{g}}
\end{aligned}
$$

For this application the scalar version of this filter is used, but a matrix extension is also proposed in the original paper [5]. In the figure [5] we can see the difference of applying the edge detector without and with the NRAD filter.
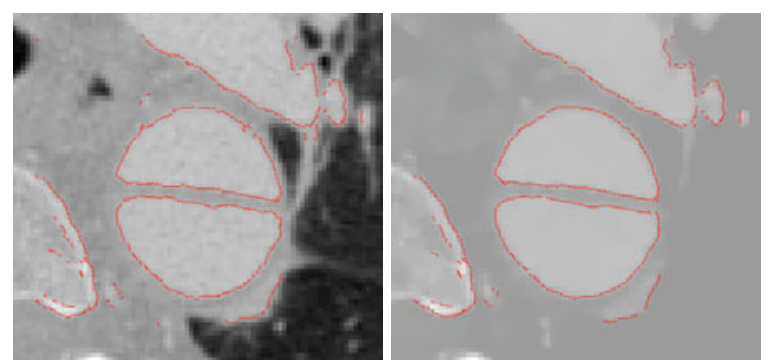

Fig. 5. Subpixel result vs Subpixel+NRAD result

\section{Results}

In order to run the experiments the opensource software AMILab (http://amilab.sourceforge.net) has been used. AMILab is a visualization and processing image software, but is also a complete crossplatform system that includes its own scripting language and a lot of algorithms, from simple operations like resizing an image, to segmentation algorithms.

If we look at an axial cut of a CTA of an aortic dissection, the aorta contains a thin black line. This line is the dissection wall. To measure the accuracy of the experiments, a set of synthetic images is used. These images are the combination of a circle and a ring, similar to an axial cut of an aortic dissection. As the features of the synthetic images (the radius of the circle and the internal and external radii of the ring) are known, the accuracy of the subpixel detector could be measured. The experiments made were: 
- The first type tests the method with different values of the thickness of the center region, our synthetic dissection.

- In the second type, we select a thickness with a low error level and add noise to the image, from 0 to 20 (standard deviation).

- Finally, we combine all the thicknesses with all the noise levels.

Figure 6a), represents the subpixel position error when varying the thickness of the ring inside the synthetic image. We can see a mean error lower than 0.2 and a standard deviation lower than 0.32 pixels. There is a big difference between a thickness of one and two pixels, because with one pixel one of the intensities near the edge is missed, and the initial hypothesis does not hold. However, the mean error remains lower than 0.2 pixels.
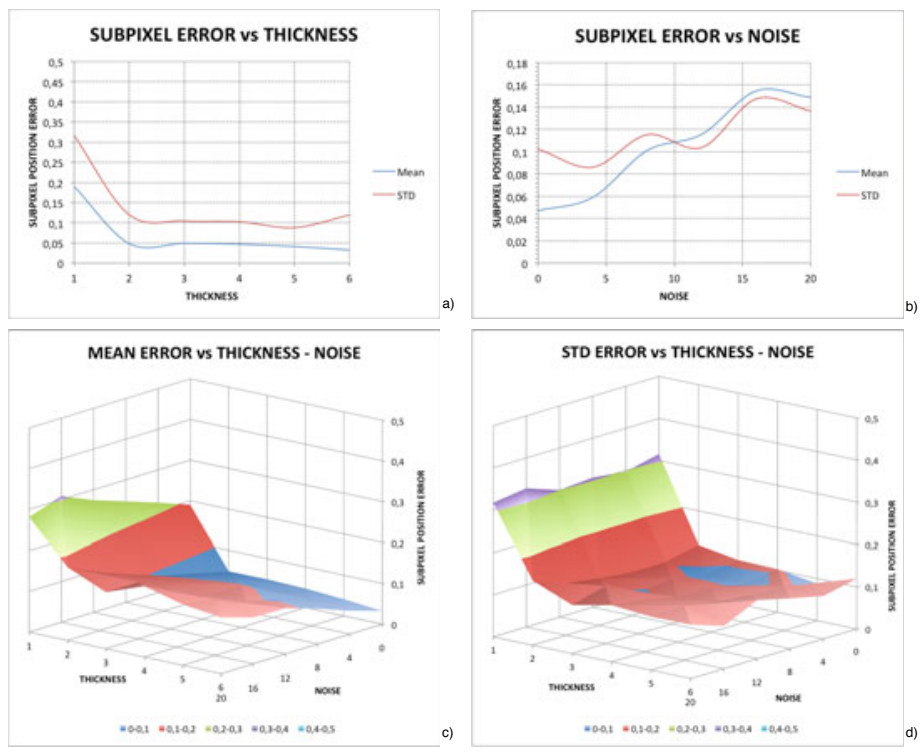

Fig. 6. Subpixel position error: a) with different thicknesses, b) with different noise levels, c) mean and d) std combining thicknesses and noise levels

Figure 6 b) represents the subpixel position error when varying the noise level on a synthetic image with a thickness of 4 . In this set of tests, the NRAD method is first applied to reduce the noise, and the subpixel method is applied to detect the edges. With the highest noise value, the mean error and standard deviation obtained are lower than 0.16 .

Finally, fig. 6] c) and d) represent the mean and the standard deviation when combining different thicknesses and noise levels. In both graphics, the error is still lower than 0.32 in the worst case (high level noise with a thickness of one). 


\section{Conclusions and Future Lines}

Throughout this paper, we have described the medical problem: its definition, classification and the images used for diagnostic. Then, the need of a subpixel level edge detector has been motivated. After describing our subpixel method, we have also added to the process the NRAD method, to reduce the noise as a preprocessing step. Then, different experiments have been designed with different dissection sizes and noise levels, showing the robustness and accuracy of our method.

From this analysis, we can conclude that the combination of the subpixel detector and the NRAD method is a very accurate process for aortic dissection detection. For example, in a pixel that meets the partial volume effect with two intensities on both sides of the edge, in the worst case, an edge detection is obtained with a maximal mean error lower than 16 percent of a pixel.

In the future, we want to incorporate the measure of the dissection wall thickness, to implement the subpixel edge detection method for 3D images (subvoxel detection) and use the subpixel information to obtain a full segmentation of the dissected aorta.

\section{References}

1. Capasso, P.: Terapéutica endovascular en la aorta torácica. In: Villamor, C., Martín, J., Moliner, M. (eds.) Diagnóstico y terapéutica endoluminal. Radiología intervencionista, pp. 303-322. Masson, Barcelona (2002)

2. DeBakey, M.E., Cooley, D.A., Creech Jr., O.: Surgical considerations of dissecting aneurysms of the aorta. Ann. Surg. 142, 586-592 (1955)

3. Da, F., Zhang, H.: Sub-pixel edge detection based on an improved moment. Image Vision Comput. 28, 1645-1658 (2010)

4. Ye, J., Fu, G., Poudel, U.P.: High-accuracy edge detection with blurred edge model. Image and Vision Computing 23(5), 453-467 (2005)

5. Krissian, K., Aja-Fernández, S.: Noise-Driven Anisotropic Diffusion Filtering of MRI. IEEE Transactions on Image Processing 18(10) (October 2009)

6. Xie, Q.L.S.H., Quin, S.: Sub-pixel edge detection for precision measurement based on canny criteria. Key Engineering Materials 295, 711-716 (2005)

7. Trujillo-Pino, A.: Localización de contornos con precisión sub-pixel en imágenes bidimensionales y tridimensionales Director: Álvarez León, L. Co-directors: Esclarín Monreal, J., Alemán Flores, M., Doctoral Thesis. Universidad de las Palmas de Gran Canaria, Computer and Systems Department (2004) 